\title{
BMJ Open Use of C-reactive protein to tailor antibiotic use: a systematic review and meta-analysis
}

\author{
Dara Petel, ${ }^{1}$ Nicholas Winters, ${ }^{2}$ Genevieve C Gore, ${ }^{3}$ Jesse Papenburg, ${ }^{4,5}$ \\ Marc Beltempo, ${ }^{6}$ Jacques Lacroix, ${ }^{7}$ Patricia S Fontela ${ }^{5,8}$
}

To cite: Petel D, Winters N, Gore GC, et al. Use of C-reactive protein to tailor antibiotic use: a systematic review and meta-analysis. BMJ Open 2018;8:e022133. doi:10.1136/ bmjopen-2018-022133

- Prepublication history and additional material for this paper are available online. To view these files, please visit the journal online (http://dx.doi. org/10.1136/bmjopen-2018022133).

Received 2 March 2018 Revised 17 October 2018 Accepted 27 November 2018

Check for updates

(c) Author(s) (or their employer(s)) 2018. Re-use permitted under CC BY-NC. No commercial re-use. See rights and permissions. Published by BMJ.

For numbered affiliations see end of article.

Correspondence to Dr Patricia S Fontela; patricia.fontela@mcgill.ca

\section{ABSTRACT}

Background and objectives C-reactive protein (CRP) has been proposed to guide the use of antibiotics. However, study results are controversial regarding the benefits of such a strategy. We synthesised the evidence of CRPbased algorithms on antibiotic treatment initiation and on antibiotic treatment duration in adults, children and neonates, as well as their safety profile.

Design Systematic review and meta-analysis.

Data sources MEDLINE, EMBASE, CENTRAL and CINAHL from inception to 20 July 2017.

Eligibility criteria for selecting studies We included randomised controlled trials (RCTs), non-RCTs and cohort studies (prospective or retrospective) investigating CRPguided antibiotic use in adults, children and neonates with bacterial infection.

Data extraction and synthesis Two researchers independently screened all identified studies and retrieved the data. Outcomes were duration of antibiotic use, antibiotic initiation, mortality, infection relapse and hospitalisation. We assessed the quality of the included studies using the Cochrane Collaboration's tool (RCTs), and A Cochrane Risk Of Bias Assessment Tool: for NonRandomized Studies of Interventions and the NewcastleOttawa scale (non-RCTs). We analysed our results using descriptive statistics and random effects models. Results Of 11165 studies screened, 15 were included. In five RCTs in adult outpatients, the risk difference for antibiotic treatment initiation in the CRP group was $-7 \%$ (95\% Cl: $-10 \%$ to $-4 \%)$, with no difference in hospitalisation rate. In neonates, CRP-based algorithms shortened antibiotic treatment duration by -1.45 days $(95 \% \mathrm{Cl}-2.61$ to -0.28$)$ in two RCTs, and by -1.15 days $(95 \% \mathrm{Cl}-2.06$ to -0.24$)$ in two cohort studies, with no differences in mortality or infection relapse.

Conclusion The use of CRP-based algorithms seems to reduce antibiotic treatment duration in neonates, as well as to decrease antibiotic treatment initiation in adult outpatients. However, further high-quality studies are still needed to assess safety, particularly in children outside the neonatal period.

PROSPERO registration number CRD42016038622

\section{INTRODUCTION}

Antibiotic resistance is an increasingly important problem worldwide, as resistant pathogens continue to emerge and few new

\section{Strengths and limitations of this study}

First meta-analysis to evaluate the use of C-reactive protein to guide antibiotic treatment decisions, as well as its safety, in adults, children and neonates.

- Use of a comprehensive search strategy and screening of a large number of studies.

- Inclusion of both interventional and observational studies which increased generalisability.

- Relatively small number of included studies for both neonatal, paediatric and adult populations.

antibiotics have been developed over the past decades. ${ }^{1-7}$ In the USA, two million cases of antibiotic-resistant infections are diagnosed annually, with more than 23000 attributable deaths. ${ }^{8}$ According to the Centers for Disease Control and Prevention, antibiotic resistance also leads to $\$ 20$ billion in excess healthcare costs, $\$ 35$ billion in societal costs and eightmillion additional hospital-days per year. ${ }^{8}$ Antibiotic overuse is a major factor contributing to the development of bacterial resistance. ${ }^{9}$ Thus, the rational use of antibiotics is critical to prevent the emergence of resistant organisms. ${ }^{10} 11$

Evidence on the optimal duration of antibiotic treatments is sparse, with many recommendations based on expert opinion. ${ }^{12} 13$ The use of infection markers such as C-reactive protein (CRP) has been proposed to improve the objectiveness of antibiotic-related decisions, including antibiotic initiation and treatment duration. CRP is an acute-phase reactant secreted in response to inflammation. ${ }^{14}$ In bacterial infections, CRP stimulates bacterial phagocytosis by binding bacterial polysaccharides and functioning as an opsonin for neutrophils and macrophages, and by activating the classical complement pathway. ${ }^{15-19}$ After the bacterial trigger for inflammation is eliminated, CRP levels decrease quickly, with a half-life of about 
19 hours. ${ }^{20-23}$ Given its physiological behaviour in bacterial infections, CRP use has been proposed to guide initiation and duration of antibiotic therapy. ${ }^{14}$ However, its effectiveness as a biomarker to guide antibiotic initiation in different settings remains controversial. Furthermore, no systematic review or meta-analysis has been performed to evaluate the benefit of using CRP to guide antibiotic treatment duration and none have been done in the neonatal or paediatric populations assessing its utility to guide antibiotic initiation. ${ }^{23-27}$

We hypothesise that a strategy based on CRP levels may safely decrease unnecessary antibiotic use for patients in whom a bacterial infection is suspected. Thus, the main objective of our systematic review and meta-analysis is to determine the effect of using a CRP-based algorithm on antibiotic consumption in patients with a suspected bacterial infection. Moreover, we aim to determine the safety of using a CRP-based strategy to guide antibiotic use.

\section{METHODS}

\section{Protocol}

We developed our protocol according to the Preferred Reporting Items for Systematic Reviews and Meta-Analyses Protocols 2015 statement. ${ }^{28}$

\section{Information sources and search strategy}

We searched Medline, MEDLINE (Ovid), EmBase (Ovid), Cochrane Central Register of Controlled Trials and CINAHL (EBSCOhost) from their inception to 20 July 2017 for eligible studies. In collaboration with a medical librarian (GG), we developed our search strategy combining search terms related to CRP and antibiotic treatment (see online supplementary materials section $1)$. Moreover, we searched for trial protocols through metaRegister (http://www.controlled-trials.com), and used Scopus for forward citation searching. We also hand searched the citations of recent reviews and included articles.

\section{Eligibility criteria}

We included original peer-reviewed articles in which CRP was used to guide decisions regarding antibiotic treatment initiation or duration. Eligible studies were randomised controlled trials (RCTs), quasi-RCTs or prospective/ retrospective cohort studies. Studies had to include a comparison group that used any combination of clinical, laboratory, radiological and microbiological findings, but not CRP, to guide treatment. Included studies evaluated adult ( $>18$ years old), paediatric ( $\geq 30$ days to $<18$ years old) and neonatal ( $<30$ days old corrected gestational age) patients in any clinical setting with suspected bacterial infection. We excluded case-control and cross-sectional studies, abstracts, literature reviews, editorials and studies not conducted in humans. Languages were restricted to English, French, Spanish, Italian and Portuguese.

\section{Interventions and outcomes}

Our primary intervention was the use of CRP levels to inform antibiotic initiation and/or duration. Our primary outcome was length of antibiotic use (number of days of antibiotic treatment received by each patient). Secondary outcomes included antibiotic initiation (proportion of patients who received antibiotic treatment), mortality and infection relapse (return of signs and symptoms related to initial infection within 2 weeks after stopping antibiotics and/or growth of at least one initial causative bacterial strain from a new culture) ${ }^{29}$

\section{Study selection}

Two reviewers (DP and NW) independently performed the first screen (title and abstract), and the full-text screen of the studies retrieved by our search. Discrepancies were resolved by consensus or by the opinion of an arbitrator (PSF).

\section{Data extraction}

Three researchers (DP, NW and PSF) created the data extraction form that was piloted with $13 \%$ of the included publications. We then modified and finalised the form. The same two reviewers independently extracted the data. We recorded data pertaining to population demographics, study design/setting, author, publication year, journal, funding sources, sample size, intervention (CRP cut-off values, type of CRP test (laboratory or point-of-care)), the aforementioned study outcomes and study quality. Detailed information on extracted variables is presented in online supplementary materials section 2 .

\section{Quality assessment}

Three trained reviewers (DP, NW and PSF) independently assessed the quality of the included studies. We used the Cochrane Collaboration's tool for assessing risk of bias in RCTs. ${ }^{30}$ The tool's items include: adequacy of randomisation and allocation concealment; blinding; completeness of outcome data; and selective reporting. Each item was graded as 'low', 'high' and 'unclear' risk of bias.

We assessed the quality of quasi-RCT and cohort studies using A Cochrane Risk Of Bias Assessment Tool: for Non-Randomized Studies of Interventions tool. ${ }^{31}$ The items included are: presence of confounding, selection bias, intervention measurement bias, bias due to departures from intended interventions, missing data, outcome measurement bias and reporting bias. Studies were graded as 'low', 'moderate', 'serious' and 'critical' risk of bias, with 'no information' used to represent missing data. Moreover, non-randomised studies were also assessed using the Newcastle-Ottawa scale which focuses on comparability and selection of study participants, and outcome ascertainment. ${ }^{32}$ This grading scale uses a 'star system' with a maximum of nine stars allotted (highest possible quality).

\section{Patient and public involvement}

No patients were involved in the development of this study. 


\section{Data synthesis and statistical analysis}

We pooled studies that were clinically comparable (ie, similar populations, designs and treatments) and assessed statistical heterogeneity using the $\mathrm{I}^{2}$ statistic. ${ }^{30}$ To estimate summary differences in the duration of antibiotic treatment between the control and CRP treatment groups, we calculated the standardised mean difference (SMD) and their $95 \%$ CIs in the number of treatment days using random effects (DerSimonian and Laird method) models. ${ }^{33}$ For studies that only reported medians, we estimated the mean and SD using the methods proposed by Wan et $a l^{35}$ For antibiotic initiation, mortality and relapse, we estimated absolute risk differences (RD) and their 95\% CI using random effects models. When assessing safety outcomes, we used non-inferiority margins of $5 \%$ for infection relapse and hospitalisation, and $2 \%$ for mortality. We stratified our analyses by patient population (adult, paediatric, neonatal), and then by study design (RCT or non-randomised). We could not assess publication bias because of the limited number of studies available. All analyses were conducted in Stata V.12 (StataCorp). ${ }^{36}$

\section{RESULTS}

We identified 11165 titles. After removal of duplicates, we screened the titles/abstracts of 8504 records and assessed the full text of 57 articles (figure 1). Of the 15 studies included in this review (table 1), 10 were RCTs, 1 was a quasi-RCT and 4 were cohort studies (two retrospective and two prospective). ${ }^{21}$ 24-27 37-46

Eight studies used CRP to guide initiation of antibiotics and six studies $(75 \%)$ included adult populations. CRP cut-offs used to guide treatment were similar across adult studies, with most studies withholding antibiotics when CRP was $<20 \mathrm{mg} / \mathrm{L}$, using discretion when CRP was between $20 \mathrm{mg} / \mathrm{L}$ and $100 \mathrm{mg} / \mathrm{L}$, and initiating treatment when CRP $>100 \mathrm{mg} / \mathrm{L}$. Comparators used in the antibiotic initiation studies were similar. Regarding infection type, all adult studies included patients with respiratory
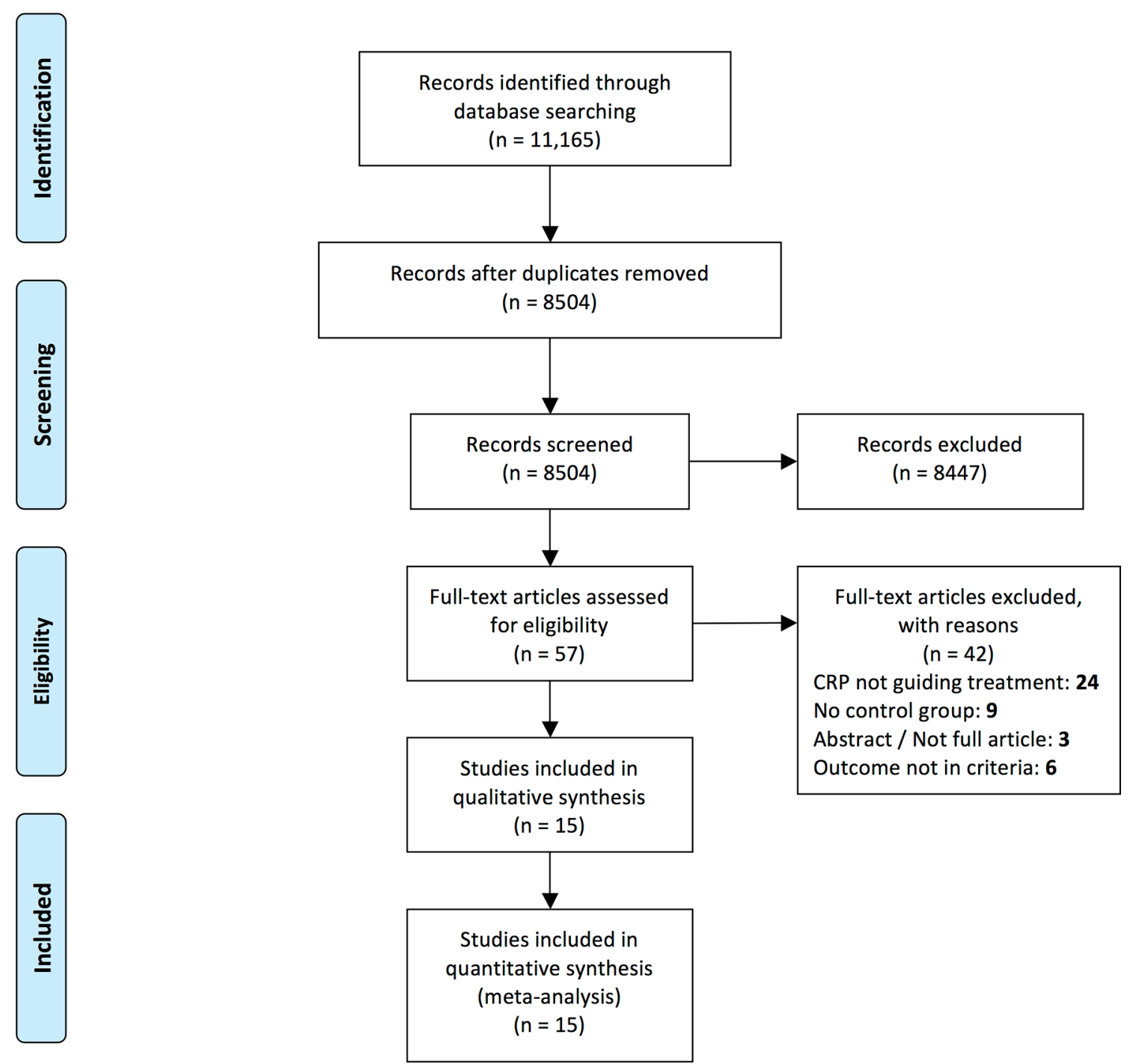

Figure 1 Flow diagram of search results and study inclusion according to the Preferred Reporting Items for Systematic Reviews and Meta-Analyses statement guidelines. CRP, C-reactive protein. 


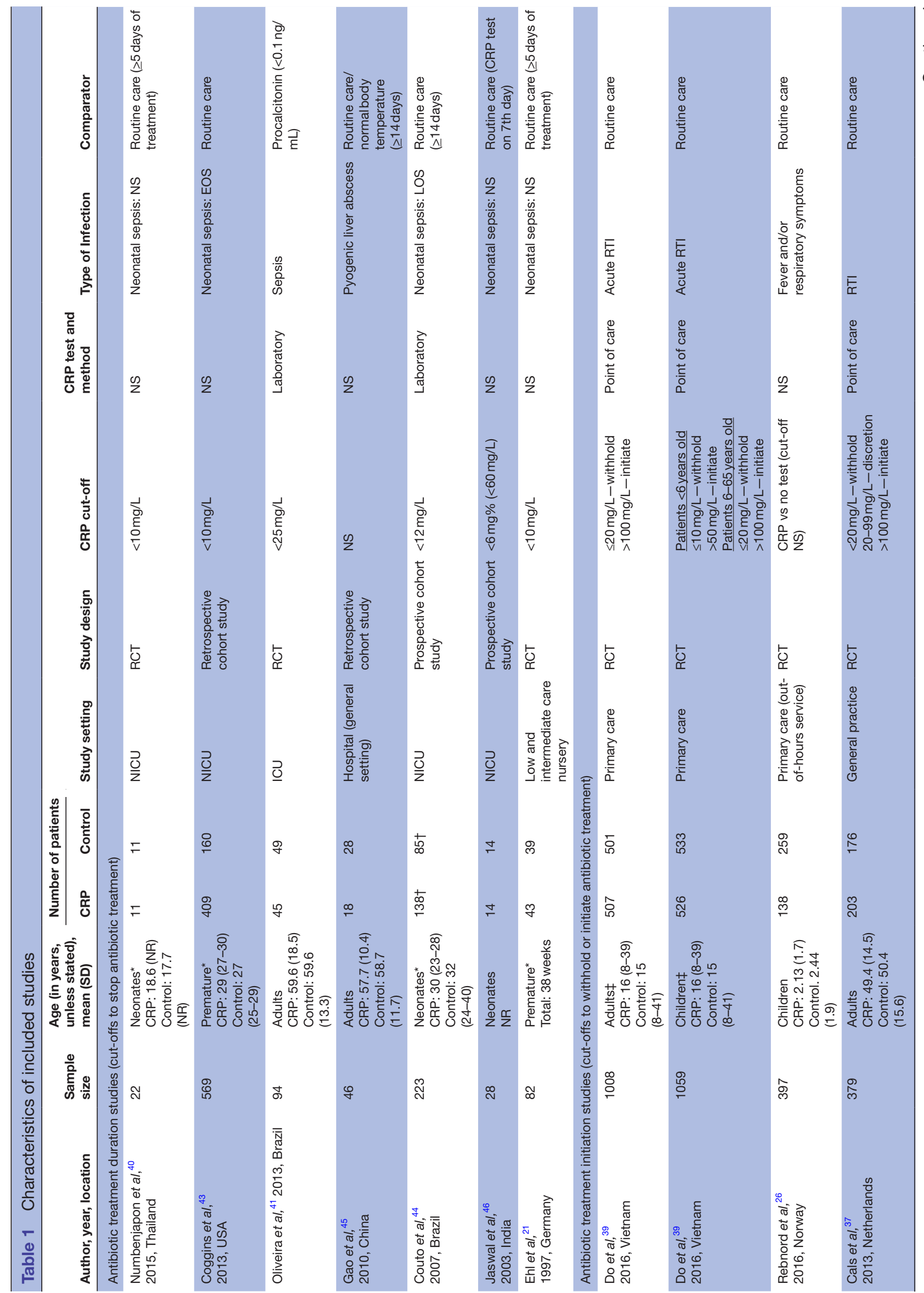




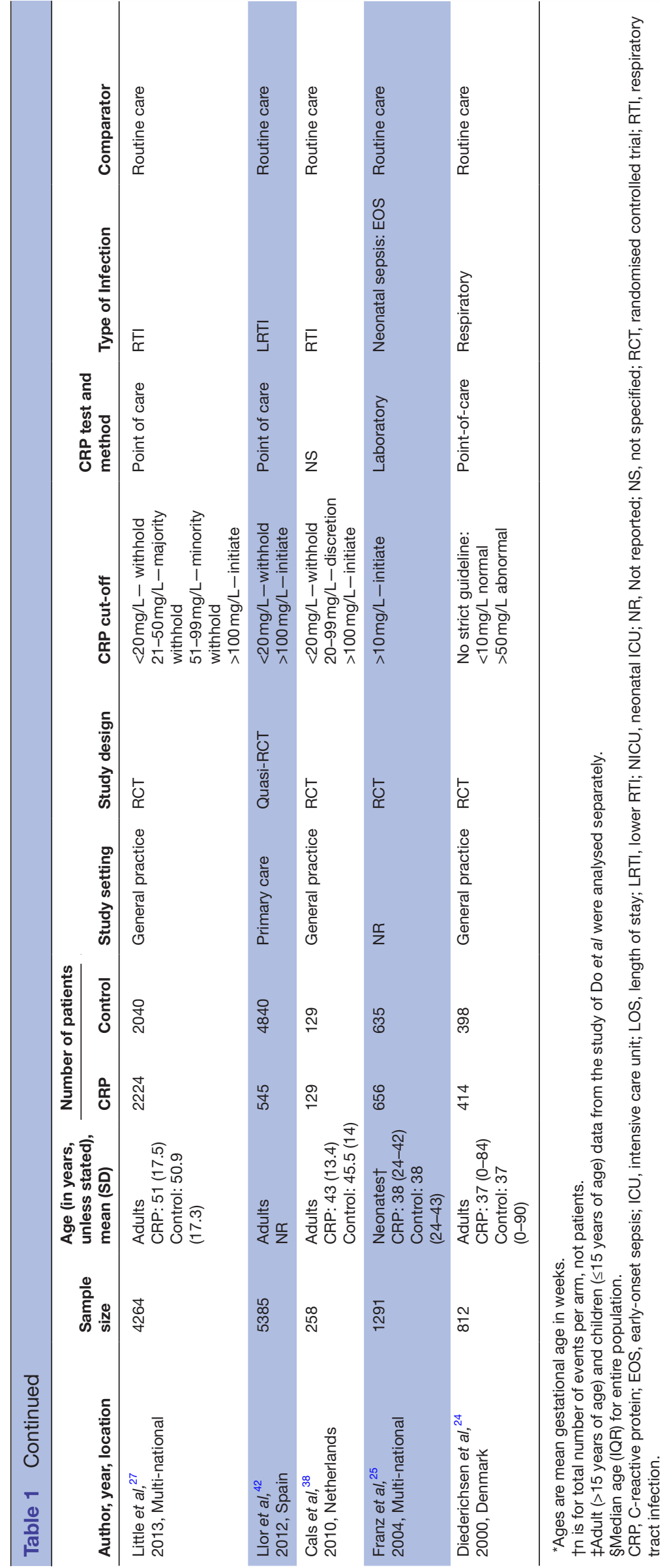

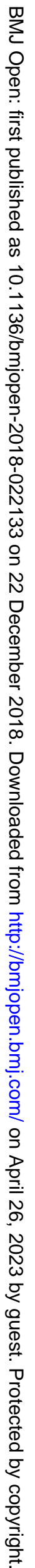




\begin{tabular}{|c|c|c|c|c|c|c|c|}
\hline First Author, Year & Study Design & $\begin{array}{c}\text { Random sequence } \\
\text { generation }\end{array}$ & $\begin{array}{c}\text { Allocation } \\
\text { concealment }\end{array}$ & $\begin{array}{c}\text { Blinding of } \\
\text { participants \& } \\
\text { personnel }\end{array}$ & $\begin{array}{c}\text { Blinding of } \\
\text { outcome } \\
\text { assessment }\end{array}$ & $\begin{array}{l}\text { Incomplete } \\
\text { outcome data }\end{array}$ & Selective reporting \\
\hline Do et al. 2016 & RCT & C & D & & & & \\
\hline Rebnard et al. 2016 & $\mathrm{RCT}$ & & & & & & \\
\hline Numbenjapon et al. 2015 & $\mathrm{RCT}$ & & & & & & \\
\hline Cals et al. 2013 & $\mathrm{RCT}$ & & & & & & \\
\hline Little et al. 2013 & RCT & & & & & & \\
\hline Oliveira et al. 2013 & $\mathrm{RCT}$ & & & & & & \\
\hline Cals et al. 2010 & $\mathrm{RCT}$ & & & & & & \\
\hline Franz et al. 2004 & $\mathrm{RCT}$ & & & & & & \\
\hline Diederichsen et al. 2000 & $\mathrm{RCT}$ & & & & & & \\
\hline Ehl et al. 1997 & $\mathrm{RCT}$ & & & & & & \\
\hline
\end{tabular}

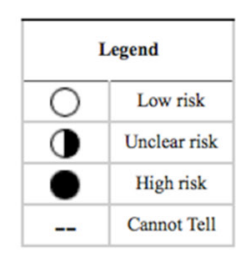

\begin{tabular}{|c|c|c|c|c|c|c|c|c|c|}
\hline First Author, Year & Study Design & $\begin{array}{c}\text { Bias due to } \\
\text { confounding }\end{array}$ & \begin{tabular}{|c|}
$\begin{array}{c}\text { Bias in selection of } \\
\text { participants into } \\
\text { the study }\end{array}$ \\
\end{tabular} & $\begin{array}{c}\text { Bias in } \\
\begin{array}{c}\text { measurement of } \\
\text { interventions }\end{array} \\
\end{array}$ & $\begin{array}{c}\text { Bias due to missing } \\
\text { data }\end{array}$ & $\begin{array}{c}\text { Bias in } \\
\text { measurement of } \\
\text { outcomes }\end{array}$ & $\begin{array}{l}\text { Bias in selection of } \\
\text { the reported result }\end{array}$ & Overall & $\begin{array}{l}\text { Newcastle- } \\
\text { Ottawa Scale }\end{array}$ \\
\hline Coggins et al. 2013 & Cohort & & & 0 & & 0 & & & 7 \\
\hline Gao et al. 2010 & Cohort & & & & & & & & 6 \\
\hline Couto et al. 2007 & Cohort & & & & & 0 & & & 9 \\
\hline Jaswal et al. 2003 & Cohort & & & 0 & & $C$ & & & 6 \\
\hline Llor et al. 2012† & Cohort & & & & & & & & \\
\hline
\end{tabular}

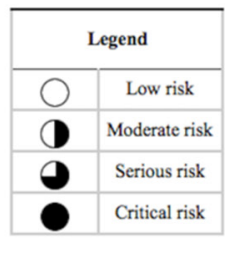

Figure 2 Quality assessment of the included studies according to Cochrane Collaboration's tools for randomised controlled trials (RCTs) and A Cochrane Risk Of Bias Assessment Tool: for Non-Randomized Studies of Interventions tool for cohort studies and quasi-RCTs.

tract infections. Details of the outcomes used in analyses are found in online supplementary materials section 3 table 1 .

We included seven studies that investigated duration of antibiotics. Their patient populations included neonates (three studies; 42\%), premature infants (two studies; $28 \%$ ) and adults (two studies; 28\%). The CRP cut-offs used to stop antibiotics were similar and ranged from $10 \mathrm{mg} / \mathrm{L}$ to $25 \mathrm{mg} / \mathrm{L}$, while one study reported a cut-off value of $6 \mathrm{mg} \%(60 \mathrm{mg} / \mathrm{L})$. The comparators used were similar across studies, and the only difference was the minimum duration of antibiotic use ( 7 days or 14 days of treatment). The type of infections varied between studied patient populations, e.g., all neonatal studies included septic patients, but the categorisation of early or late sepsis was inconsistent.

\section{Quality of included studies}

Figure 2 shows the results of the quality assessment of included studies. Most RCTs presented low risk of bias regarding randomisation and allocation concealment. However, in seven $(70 \%)$ of the included RCTs, the authors were unable to either blind the participants and personnel or blind the assessment of the outcome which led to a high risk of bias for this criterion. Furthermore, we could not assess selective reporting because only three (20\%) studies had protocols either registered or published. However, no evidence was found within the included studies to indicate that such bias was present. Overall, the included cohort studies were at moderate to serious risk of bias, primarily due to confounding and selection bias, and no studies were at critical risk of bias in any category. According to the Newcastle-Ottawa scale, the mean ranking of the four cohort studies was 7 (out of 9) stars.

\section{Use of CRP to guide initiation of antibiotics}

Eight studies investigated the use of CRP to guide antibiotic treatment initiation. The pooled RD for initiation of antibiotics from five RCTs conducted in adult populations (figure 3 ) was $-7 \%(95 \% \mathrm{CI}-10 \%$ to $-4 \%)$, and the statistical heterogeneity between studies was moderate $\left(\mathrm{I}^{2}=38 \%\right)$. We also preformed a sensitivity analysis by removing Little et al s RCT that led to comparable results (RD $-7 \% ; 95 \% \mathrm{CI}-11 \%$ to $-2 \%$ ). Similar results were observed in one cohort study in adults (RD $-8 \%$; $95 \%$ CI $-11 \%$ to $-4 \%)$. $^{42}$

Regarding neonates, in one RCT the estimated reduction in the absolute risk of initiating antibiotics was $7 \%$ $(95 \%$ CI $-11 \%$ to $-2 \%) .{ }^{25}$ Finally, two RCTs including children indicated no difference between CRP and control groups (RD $-3 \%$; $95 \% \mathrm{CI}-14 \%$ to $8 \%$ ). ${ }^{2639}$

\section{Use of CRP to guide duration of antibiotic use}

We stratified our analyses by population and study design, as these categorisations provided a greater clinical homogeneity of pooled data. After combining the two RCTs including neonatal and premature patients (figure 4), the SMD for duration of antibiotic use was -1.45 days (95\% CI -2.61 to -0.28$)$. The pooled SMD for duration of antibiotic treatment from two neonatal cohort studies was -1.15 days $(95 \%$ CI -2.06 to -0.24$)$. Despite the low clinical heterogeneity between the studies, the statistical heterogeneity for the pooled estimates from RCTs and cohorts was substantial $\left(\mathrm{I}^{2}=75.7 \%\right.$ and $96.4 \%$, respectively).

Only one RCT and one cohort study were conducted in adult populations; both showed a reduction in duration of antibiotic use. In the study by Oliveira et al, the difference was -0.25 days $(95 \%$ CI -0.66 to 0.16$) .{ }^{41}$ Meanwhile, in the cohort study by Gao et al, the SMD was -1.10 days $(95 \%$ CI -1.74 to -0.47$) .{ }^{45}$ No paediatric studies 


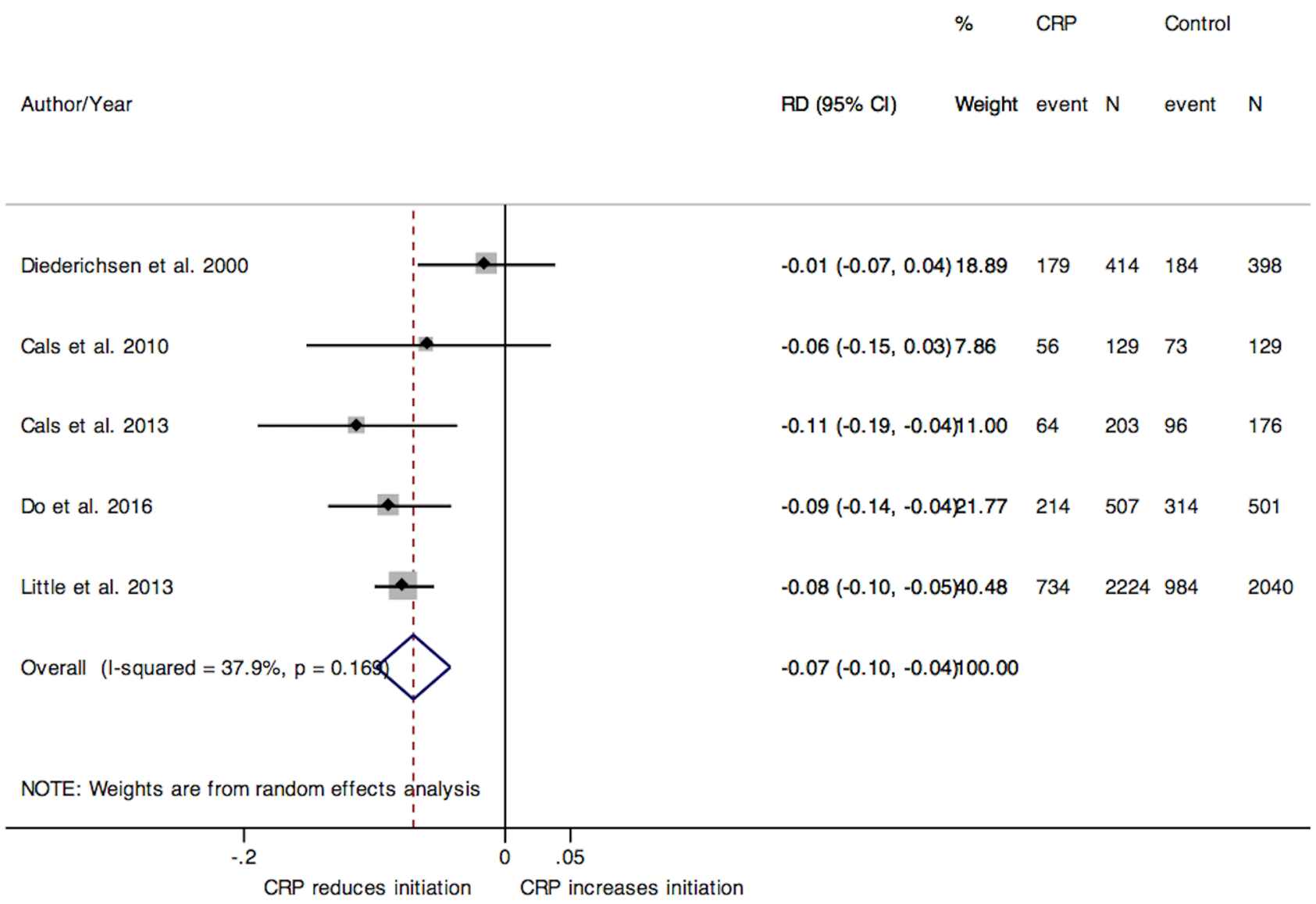

Figure 3 Forest plot of the pooled RD for adult RCTs on antibiotic use initiation. CRP, C-reactive protein; RCT, randomised controlled trial; $\mathrm{RD}$, risk difference.

evaluating CRP use to guide antibiotic treatment duration were retrieved.

\section{Mortality}

In the studies of neonates and premature populations where CRP was used to guide duration of antibiotic treatment, the pooled RD for hospital mortality from two RCTs and from two cohort studies was $0 \%(95 \% \mathrm{CI}-4$ to 4$)$ and $-5 \%$ (95\% CI -10 to 0$)$, respectively (figure 5$).{ }^{21404344} \mathrm{In}$ the single RCT conducted in adults, there was no difference between treatment groups regarding mortality (RD $2 \%$; $95 \%$ CI -14 to 17$){ }^{41}$ No deaths were observed in adult studies where CRP was used to guide antibiotic initiation (online supplementary materials section 4 figure 1). ${ }^{27}{ }^{37-39}$ No paediatric studies evaluating CRP use and mortality were retrieved.

\section{Infection relapse}

Data regarding relapse were only reported in studies where CRP was used to guide treatment duration. For studies of neonates and premature populations, the pooled RD for relapse between treatment groups from two RCTs was $-4 \%$ (95\% CI $-12 \%$ to $3 \%)$ and from two cohort studies was $-1 \%(95 \% \mathrm{CI}-4 \%$ to $3 \%)$, as seen in figure $6 .{ }^{21} 404446$ One RCT and one cohort conducted in adult populations both indicated no difference in relapse between groups (data not shown). ${ }^{41}{ }^{45}$ We did not retrieve paediatric studies evaluating CRP use and infection relapse.

\section{Hospitalisation}

Data for hospitalisations were only reported in adult outpatient studies where CRP was used to guide antibiotic initiation. From four RCTs, the pooled RD for hospitalisation between treatment groups was $0 \%(95 \%$ CI $-0.00 \%$ to $0.01 \%$ ) as can be seen in supplementary materials section 4 -figure $2 .^{37-39}$

\section{DISCUSSION}

This systematic review and meta-analysis showed that the use of CRP-driven antibiotic therapy was associated with a decreased duration of antibiotic use in neonatal patients. Similarly, CRP-based algorithms also reduced antibiotic initiation in adult outpatients. The above findings were consistent regardless of the varied designs of included studies in this review and also diversity of the study populations which come from both high and low-income countries. Thus, based on our results, the recommended CRP cut-off for antibiotic treatment stopping in newborns with neonatal sepsis is $<10 \mathrm{mg} / \mathrm{L}$. In adult outpatients with respiratory tract infections, the recommended CRP cut-offs for antibiotic withholding and treatment initiation are $<20 \mathrm{mg} / \mathrm{L}$ and $\geq 100 \mathrm{mg} / \mathrm{L}$, respectively. 


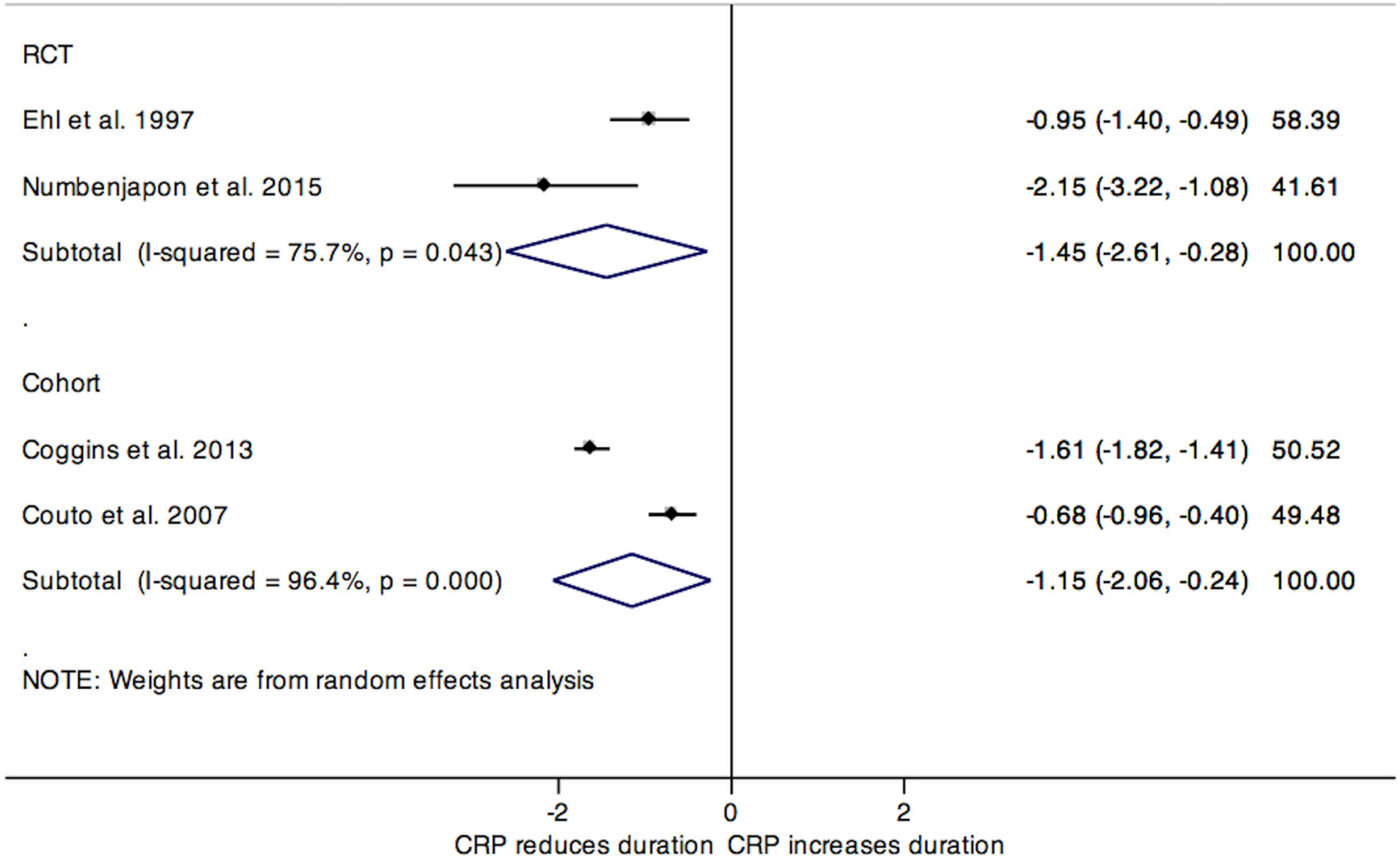

Figure 4 Forest plot for the SMD in duration of antibiotic use in studies of neonates and premature populations, stratified by study design. CRP, C-reactive protein; RCT, randomised controlled trial; SMD, standardised mean differences.

Importantly, the use of CRP algorithms to guide antibiotic treatment appears to be safe, as neonatal studies using CRP to determine duration of antibiotic treatment showed no difference in mortality or in infection relapse. Furthermore, adult studies that used CRP to guide antibiotic initiation showed no differences in mortality and hospitalisation rates.

CRP is an acute-phase reactant synthetised mainly in the liver, but also by macrophages and lymphocytes, and secreted in plasma in response to inflammation, infection, tissue damage and malignancy. ${ }^{15}$ Its secretion is regulated by cytokines, with levels beginning to rise 6 hours after the initial stimulus and reaching a peak in 48 hours. ${ }^{15} 20$ During infection, CRP stimulates bacterial phagocytosis by binding bacterial polysaccharides and functioning as an opsonin for neutrophils and macrophages, and by activating the classical complement pathway. ${ }^{15-19}$ Once the trigger for inflammation is eliminated, CRP is catabolised by hepatocytes and rapidly cleared from circulation. ${ }^{20-23}$ In healthy adults, the median CRP concentration is $1.5 \mathrm{mg} / \mathrm{L}$, with levels above $100 \mathrm{mg} / \mathrm{L}$ being associated with bacterial infections. ${ }^{47-49}$

In healthy term neonates, CRP normal levels depend mainly on postnatal age, with median levels gradually increasing from birth $(0.4 \mathrm{mg} / \mathrm{L})$ to 48 hours post partum $(2.7 \mathrm{mg} / \mathrm{L})$, and then declining at 96 hours $(1.4 \mathrm{mg} / \mathrm{L}){ }^{50}{ }^{51}$ Importantly, CRP values above $10 \mathrm{mg} / \mathrm{L}$, the cut-off most often used to diagnose neonatal sepsis, are not uncommonly observed during the first 72 hours after birth which may jeopardise its utility for the diagnosis of early-onset sepsis and, consequently, to determine the appropriateness of antibiotic treatment initiation in this population. ${ }^{50}{ }^{51}$ This may also partially explain the great variability in CRP sensitivity ( $30 \%$ to $80 \%$ ) to diagnose neonatal sepsis for cut-offs between $4 \mathrm{mg} / \mathrm{L}$ and $15 \mathrm{mg} / \mathrm{L}$ observed in Hedegaard et $a l$ s systematic review. ${ }^{52}$ Given the suboptimal diagnostic performance of CRP in a patient population with high mortality risk due to sepsis, it is not surprising that our meta-analysis showed inconclusive results regarding antibiotic treatment initiation in neonates.

We demonstrated that the use of CRP decreases antibiotic treatment duration in full-term and premature newborns. Nevertheless, the question about a potential difference in the performance of CRP to guide antibiotic duration in early-onset versus late-onset sepsis remains. As included studies used different sepsis definitions, it was not possible to address this limitation. This may be important because early-onset sepsis is mainly caused by Gram-negative bacilli and group B streptococcus 


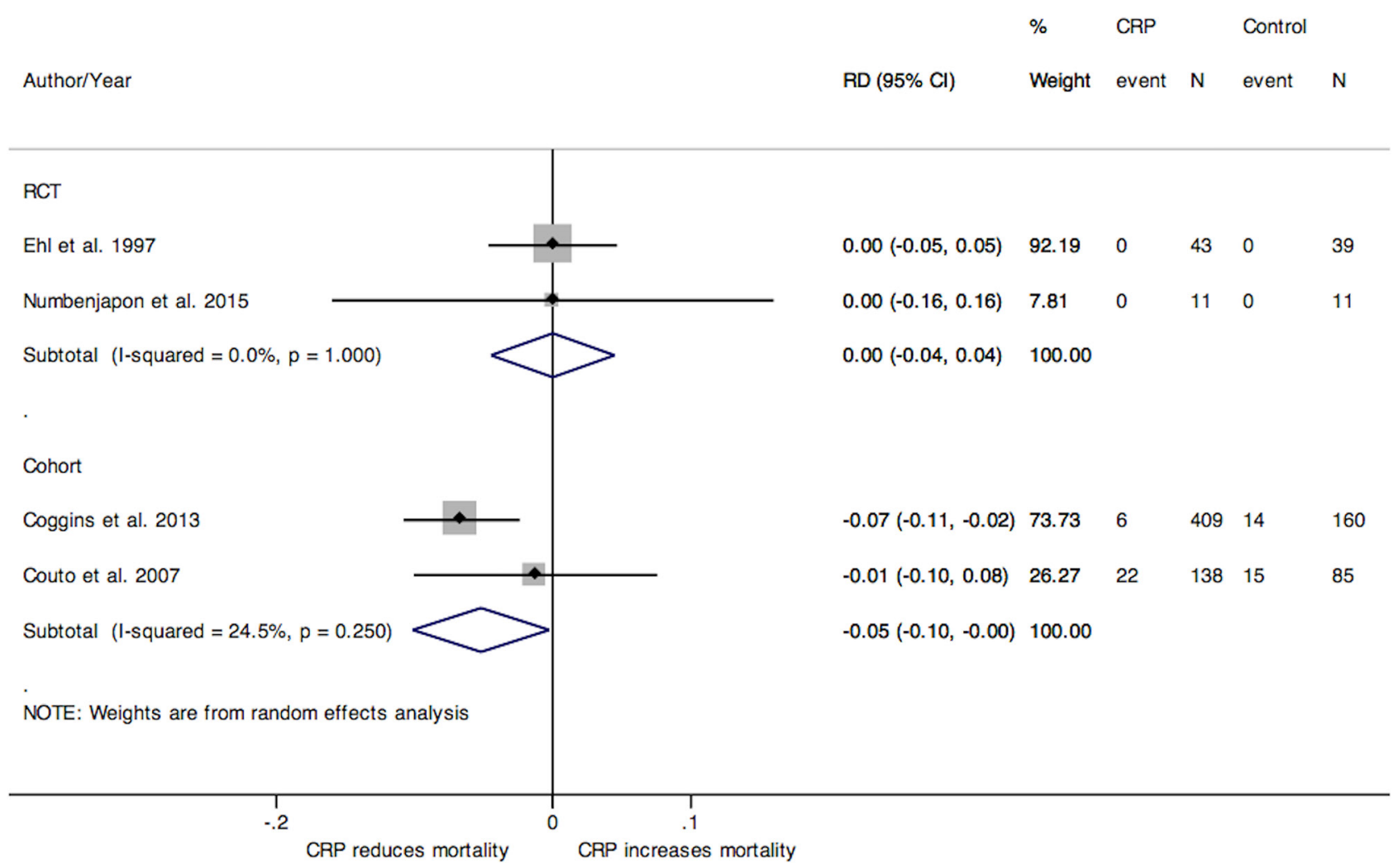

Figure 5 Forest plot of the RD for mortality between CRP treatment and control groups from studies investigating the duration of antibiotic use in neonates and premature populations, stratified by study design. CRP, C-reactive protein; RCT, randomised controlled trial; RD, risk difference.

which typically provoke a much stronger host inflammatory response than the coagulase-negative Staphylococci frequently associated with late-onset sepsis. ${ }^{53}$ Thus, the ability of CRP to guide antibiotic use may differ across these two scenarios.

The diagnostic performance of CRP for respiratory tract infections in adult patients was evaluated in different meta-analyses. Falk et al showed that at CRP cut-off $\leq 20 \mathrm{mg} / \mathrm{L}$, the pooled positive and negative likelihood ratios were 2.1 (95\% CI 1.8 to 2.4 ) and 0.33 (95\% CI 0.21 to 0.53 ), respectively. ${ }^{54}$ Furthermore, the individual patient data meta-analysis of Minnaard et al including 5308 subject showed that the addition of CRP to a pneumonia prediction model improves its discriminatory ability, with a pooled improvement of the area under the curve of 0.075 (95\% CI 0.04 to 0.11$){ }^{55}$

Regarding the effect of CRP on antibiotic use for respiratory tract infections, the reduction in antibiotic initiation observed in our study is well aligned with Huang $e t$ $a l$ s meta-analysis results. ${ }^{56}$ In that study, the use of point-of care CRP was associated with a reduction in antibiotic prescription (relative risk $0.75 ; 95 \% \mathrm{CI} 0.67$ to 0.83 ) at the index consultation for adult outpatients with respiratory tract infections. The low risk of morbidity and mortality associated with such infections allows physicians to use a 'wait and see' approach. However, differently from the aforementioned meta-analyses, our study showed that CRP-based algorithms also reduce antibiotic treatment duration, with no increase in hospitalisation rates. The latter outcome is essential to evaluate the safety of CRP to guide antibiotic use.

Our meta-analysis showed that the use of CRP-based algorithms to determine antibiotic treatment duration did not impact infection relapse in neonates. This is important since the prolonged use of antibiotics in infants without culture-proven infection has been associated with higher risk of mortality or morbidity. ${ }^{57}$ However, while the non-inferiority margin for mortality of cohort studies was $0 \%$, the non-inferiority margin of the two included RCTs was $5 \%$. The heterogeneity of such results, due to the relatively small sample sizes of the RCTs $(n=82$ and $\mathrm{n}=22),{ }^{21}{ }^{40}$ demonstrates the need for further studies of larger sample size to evaluate the safety of using CRP based algorithms to guide antibiotic treatment duration for these patients.

Regarding adult patients, no deaths were observed and hospitalisation rates were similar (by a non-inferiority margin of $1 \%$ ) in adult studies that used CRP to guide antibiotic initiation. Nevertheless, the non-inferiority margin for mortality in the study ${ }^{41}$ evaluating the use of CRP algorithms to guide duration of antibiotic treatment in this patient population was $18 \%$ which breaches any reasonable non-inferiority margin to determine safety. Finally, due to the low number of deaths and relapses observed in neonates and adults, we should interpret the aforementioned safety results with caution.

There is scarce literature comparing the performance of CRP to other biomarkers to guide antibiotic use. The 


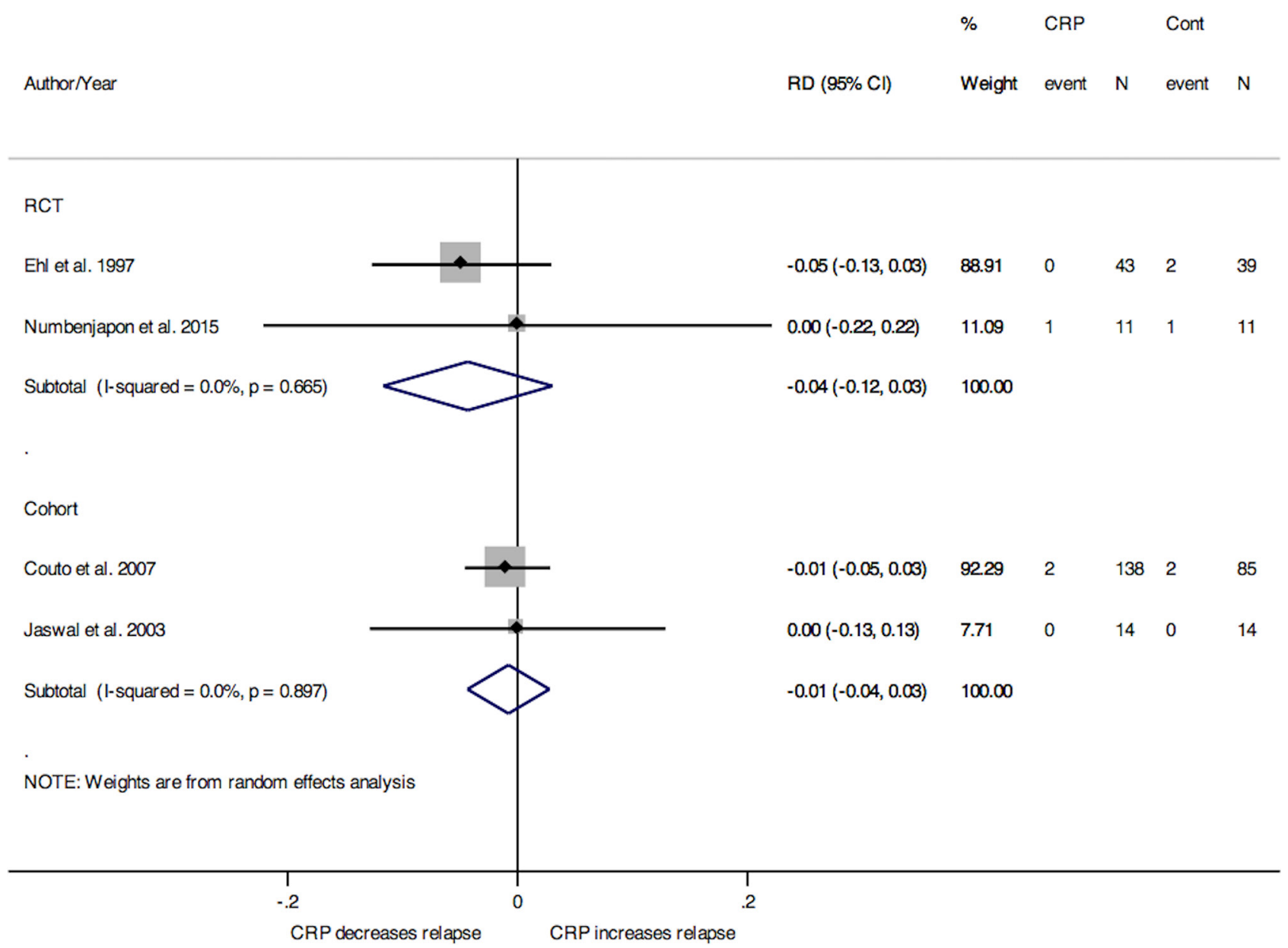

Figure 6 Forest plot for the risk differences $(\mathrm{RD})$ in infection relapse between treatment and control group for studies investigating duration of antibiotic use in neonates and premature populations, stratified by study design. CRP, C-reactive protein; $\mathrm{RCT}$, randomised controlled trial; $\mathrm{RD}$, risk difference.

RCT of Oliveira et al comparing the use of CRP and procalcitonin algorithms to determine antibiotic treatment duration included 94 critically ill adult patients with severe sepsis or septic shock. No difference in the median duration of treatment was observed between the procalcitonin (7 days; IQR 6-8.5) and CRP groups (6 days; IQR 5-7).$^{58}$ Importantly, the study treatment algorithm imposed an upper limit of 7 days of antibiotic treatment for patients who showed signs of clinical resolution of sepsis, independently of CRP and procalcitonin levels which may have contributed for the lack of difference between groups.

Our study presents limitations. The relatively small number of included studies, both for neonatal, paediatric and adult populations, limited our ability to interpret and generalise our results and lessened their precision. The overall quality of the included RCTs was affected by the inability to blind participants, while quality of included cohort studies overall appeared slightly better. Moreover, we were unable to assess the presence of selective reporting, as many RCTs did not provide original study protocols; however, we do not suspect that this was an important issue in the included studies. Finally, there were no data available on the use of CRP to guide antibiotic treatment duration in paediatric patients. It is possible that CRP cut-offs and performance are not the same in children, as their baseline cytokine levels are higher compared with neonates. ${ }^{59-62}$

Nevertheless, our study also has important strengths. It is the first meta-analysis to explore the use of CRP to guide antibiotic treatment duration. We succeeded in using a comprehensive search strategy to retrieve and screen a very large number of articles, including both interventional and observational studies. The inclusion of both study designs allowed for a realistic analysis of CRP use in conditions representative of clinical practice. Importantly, although there was high statistical heterogeneity between studies, they were clinically homogeneous which led to our decision of performing a meta-analysis.

In summary, CRP-guided treatment decreases the duration of antibiotic treatment in neonates. Antibiotic initiation and treatment duration were also reduced in adult outpatients when CRP was used. This practice appears to be safe, as rates of infection relapse, hospitalisations and mortality did not differ between study groups. However, due to the small number of included studies, further evaluations, mainly high-quality RCTs, are still necessary to 
definitively establish the safety and efficacy of CRP-guided algorithms.

\section{Author affiliations}

${ }^{1}$ Department of Pediatrics, University of Western Ontario, London, Ontario, Canada

${ }^{2}$ Department of Epidemiology, Biostatistics and Occupational Health, McGill

University, Montreal, Quebec, Canada

${ }^{3}$ Schulich Library of Physical Sciences, Life Sciences and Engineering, Montreal,

Canada

${ }^{4}$ Division of Pediatric Infectious Diseases, Department of Pediatrics, McGill

University, Montreal, Quebec, Canada

${ }^{5}$ Department of Epidemiology, Biostatistics and Occupational Health, McGill

University, Montreal, Quebec, Canada

${ }^{6}$ Division of Neonatology, Department of Pediatrics, McGill University, Montreal,

Quebec, Canada

${ }^{7}$ Division of Pediatric Critical Care, Department of Pediatrics, Université de Montréal,

Montreal, Quebec, Canada

${ }^{8}$ Division of Pediatric Critical Care, Department of Pediatrics, McGill University,

Montreal, Quebec, Canada

Contributors All authors have made significant contributions to the study conception and design, article revision and have given final approval for the submitted version. The specific contributions of each author are the following: DP: study design and conduct, development of search strategy, data collection, data analysis, manuscript writing. NW: study design and conduct, data analysis manuscript writing. GCG: development of search strategy, conduct of electronic database search. JP, MB and JL: study design, data analysis, manuscript review. PSF (guarantor): study design and conduct, development of search strategy, data analysis, manuscript writing

Funding The authors have not declared a specific grant for this research from any funding agency in the public, commercial or not-for-profit sectors.

Competing interests JP reports personal fees or research grant funding from BD Diagnostic Systems, Cepheid, AbbVie and RPS Diagnostics outside the submitted work. The remaining authors have disclosed that they do not have any potential conflicts of interest.

Patient consent for publication Not required.

Provenance and peer review Not commissioned; externally peer reviewed.

Data sharing statement All data were collected from previously published research. Our dataset is available on request from the corresponding author.

Open access This is an open access article distributed in accordance with the Creative Commons Attribution Non Commercial (CC BY-NC 4.0) license, which permits others to distribute, remix, adapt, build upon this work non-commercially, and license their derivative works on different terms, provided the original work is properly cited, appropriate credit is given, any changes made indicated, and the use is non-commercial. See: http://creativecommons.org/licenses/by-nc/4.0/.

\section{REFERENCES}

1. Grant J, Saxinger L, Patrick D. Surveillance of antimicrobial resistance and antimicrobial utilization in Canada. Winnipeg, Canada: National Collaborating Centre for Infectious Diseases, 2014

2. Nelson R. Antibiotic development pipeline runs dry. New drugs to fight resistant organisms are not being developed, experts say. Lancet 2003;362:1726-7.

3. Norrby SR, Nord CE, Finch R. European Society of Clinical Microbiology and Infectious Diseases. Lack of development of new antimicrobial drugs: a potential serious threat to public health. Lancet Infect Dis 2005;5:115-9.

4. Olofsson SK, Cars O. Optimizing drug exposure to minimize selection of antibiotic resistance. Clin Infect Dis 2007;45 Suppl 2:S129-S136.

5. WHO. Antimicrobial resistance: global report on surveillance. France: World Health Organization, 2014.

6. Projan SJ. Why is big Pharma getting out of antibacterial drug discovery? Curr Opin Microbiol 2003;6:427-30.

7. Spellberg B, Powers JH, Brass EP, et al. Trends in antimicrobial drug development: implications for the future. Clin Infect Dis 2004;38:1279-86.
8. Centers for Disease Control and Prevention. Antibiotic resistance threats in the United States,2013. United States: Centers for Disease Control and Prevention, 2013.

9. Nicolini G, Sperotto F, Esposito S. Combating the rise of antibiotic resistance in children. Minerva Pediatr 2014;66:31-9.

10. Toltzis P, Rosolowski B, Salvator A. Etiology of fever and opportunities for reduction of antibiotic use in a pediatric intensive care unit. Infect Control Hosp Epidemiol 2001;22:499-504.

11. Briassoulis G, Natsi L, Tsorva A, et al. Prior antimicrobial therapy in the hospital and other predisposing factors influencing the usage of antibiotics in a pediatric critical care unit. Ann Clin Microbiol Antimicrob 2004;3:4.

12. Dellinger RP, Levy MM, Rhodes $A$, et al. Surviving sepsis campaign: international guidelines for management of severe sepsis and septic shock: 2012. Crit Care Med 2013;41:580-637.

13. Tunkel AR, Hartman BJ, Kaplan SL, et al. Practice guidelines for the management of bacterial meningitis. Clin Infect Dis 2004;39:1267-84.

14. Fontela PS, O'Donnell S, Papenburg J. Can biomarkers improve the rational use of antibiotics? Curr Opin Infect Dis 2018:31:1.

15. Ansar W, Ghosh S. C-reactive protein and the biology of disease. Immunol Res 2013;56:131-42.

16. Marnell LL, Mold C, Volzer MA, et al. C-reactive protein binds to FC gamma RI in transfected COS cells. J Immunol 1995;155:2185-93.

17. Kilpatrick JM, Volanakis JE. Opsonic properties of C-reactive protein Stimulation by phorbol myristate acetate enables human neutrophils to phagocytize C-reactive protein-coated cells. J Immunol 1985;134:3364-70.

18. Mortensen RF, Osmand AP, Lint TF, et al. Interaction of C-reactive protein with lymphocytes and monocytes: complement-dependent adherence and phagocytosis. J Immunol 1976;117:774-81.

19. Kaplan $\mathrm{MH}$, Volanakis JE. Interaction of C-reactive protein complexes with the complement system. J Immunol 1974;112:2135-47.

20. Pepys M. Hirschfield G. C-reactive protein: a critical update. J Clin Invest 2003;111:1805-12.

21. Ehl S, Gering B, Bartmann P, et al. C-reactive protein is a useful marker for guiding duration of antibiotic therapy in suspected neonatal bacterial infection. Pediatrics 1997;99:216-21.

22. Luna CM. C-reactive protein in pneumonia: let me try again. Chest 2004;125:1192-5.

23. Al-Zwaini EJ. C-reactive protein: a useful marker for guiding duration of antibiotic therapy in suspected neonatal septicaemia? East Mediterr Health J 2009;15:269-75.

24. Diederichsen HZ, Skamling M, Diederichsen A, et al. Randomised controlled trial of CRP rapid test as a guide to treatment of respiratory infections in general practice. Scand J Prim Health Care 2000;18:39-43.

25. Franz AR, Bauer K, Schalk A, et al. Measurement of interleukin 8 in combination with $\mathrm{C}$-reactive protein reduced unnecessary antibiotic therapy in newborn infants: a multicenter, randomized, controlled trial. Pediatrics 2004;114:1-8.

26. Rebnord IK, Sandvik H, Mjelle AB, et al. Out-of-hours antibiotic prescription after screening with $C$ reactive protein: a randomised controlled study. BMJ Open 2016;6:e011231.

27. Little $\mathrm{P}$, Stuart $\mathrm{B}$, Francis $\mathrm{N}$, et al. Effects of internet-based training on antibiotic prescribing rates for acute respiratory-tract infections: a multinational, cluster, randomised, factorial, controlled trial. Lancet 2013;382:1175-82.

28. Shamseer L, Moher D, Clarke M, et al. Preferred reporting items for systematic review and meta-analysis protocols (PRISMA-P) 2015: elaboration and explanation. BMJ 2015;349:97647.

29. Bouadma L, Luyt CE, Tubach F, et al. Use of procalcitonin to reduce patients' exposure to antibiotics in intensive care units (PRORATA trial): a multicentre randomised controlled trial. Lancet 2010;375:463-74.

30. Higgins J, Green S, eds. Cochrane Handbook for Systematic Reviews of Interventions Version 5.1.0 (updated March 2011): The Cochrane Collaboration, 2011.

31. Sterne J, Higgins J, Reeves B. A Cochrane Risk Of Bias Assessment Tool: for Non-Randomized Studies of Interventions (ACROBAT-NRSI), Version 1.0.0, 24, 2014.

32. Wells GA, Shea B, O'Connell D, et al. The Newcastle-Ottawa Scale (NOS) for assessing the quality of nonrandomised studies in metaanalyses. http://www.ohri.ca/programs/clinical_epidemiology/oxford. asp.

33. DerSimonian R, Laird N. Meta-analysis in clinical trials. Control Clin Trials 1986;7:177-88

34. Mantel N, Haenszel W. Statistical aspects of the analysis of data from retrospective studies of disease. J Natl Cancer Inst 1959;22:719-48. 
35. Wan X, Wang W, Liu J, et al. Estimating the sample mean and standard deviation from the sample size, median, range and/or interquartile range. BMC Med Res Methodol 2014;14:135.

36. StataCorp. Stata Statistical Software: Release 12. TX: StataCorp LP, 2011.

37. Cals JW, de Bock L, Beckers PJ, et al. Enhanced communication skills and C-reactive protein point-of-care testing for respiratory tract infection: 3.5-year follow-up of a cluster randomized trial. Ann Fam Med 2013;11:157-64.

38. Cals JW, Schot MJ, de Jong SA, et al. Point-of-care C-reactive protein testing and antibiotic prescribing for respiratory tract infections: a randomized controlled trial. Ann Fam Med 2010;8:124-33

39. Do NT, Ta NT, Tran NT, et al. Point-of-care C-reactive protein testing to reduce inappropriate use of antibiotics for non-severe acute respiratory infections in Vietnamese primary health care: a randomised controlled trial. Lancet Glob Health 2016;4:e633-41.

40. Numbenjapon N, Chamnanwanakij S, Sangaroon P, et al. C-reactive protein as a single useful parameter for discontinuation of antibiotic treatment in Thai neonates with clinical sepsis. J Med Assoc Thai 2015;98:352-7.

41. Oliveira CF, Botoni FA, Oliveira CRA, et al. Procalcitonin versus c-reactive protein for guiding antibiotic therapy in sepsis. Crit Care Med 2013;41:2336-43.

42. Llor C, Cots JM, López-Valcárcel BG, et al. Interventions to reduce antibiotic prescription for lower respiratory tract infections: Happy Audit study. Eur Respir J 2012;40:436-41.

43. Coggins SA, Wynn JL, Hill ML, et al. Use of a computerized C-reactive protein (CRP) based sepsis evaluation in very low birth weight (VLBW) infants: a five-year experience. PLoS One 2013;8:e78602

44. Couto RC, Barbosa JA, Pedrosa TM, et al. C-reactive protein-guided approach may shorten length of antimicrobial treatment of cultureproven late-onset sepsis: an intervention study. Braz J Infect Dis 2007;11:240-5.

45. Gao HN, Yuan WX, Yang MF, et al. Clinical significance of C-reactive protein values in antibiotic treatment for pyogenic liver abscess. World J Gastroenterol 2010;16:4871-5

46. Jaswal RS, Kaushal RK, Goel A, et al. Role of C-reactive protein in deciding duration of antibiotic therapy in neonatal septicemia. Indian Pediatr 2003;40:880-3.

47. Shine $B$, de Beer FC, Pepys MB. Solid phase radioimmunoassays for human C-reactive protein. Clin Chim Acta 1981;117:13-23.

48. Morley JJ, Kushner I. Serum C-reactive protein levels in disease. Ann NY Acad Sci 1982;389:406-18.
49. Rifai N, Ridker PM. Population distributions of C-reactive protein in apparently healthy men and women in the United States: implication for clinical interpretation. Clin Chem 2003;49:666-9.

50. Chiesa $\mathrm{C}$, Natale F, Pascone $\mathrm{R}$, et al. $\mathrm{C}$ reactive protein and procalcitonin: reference intervals for preterm and term newborns during the early neonatal period. Clin Chim Acta 2011;412:1053-9.

51. Perrone $\mathrm{S}$, Lotti $\mathrm{F}$, Longini $\mathrm{M}$, et al. $\mathrm{C}$ reactive protein in healthy term newborns during the first 48 hours of life. Arch Dis Child Fetal Neonatal Ed 2018;103.

52. Hedegaard SS, Wisborg K, Hvas A-M. Diagnostic utility of biomarkers for neonatal sepsis - a systematic review. Infect Dis 2015;47:117-24.

53. Shane AL, Sánchez PJ, Stoll BJ. Neonatal sepsis. Lancet 2017;390:1770-80

54. Falk G, Fahey T. C-reactive protein and community-acquired pneumonia in ambulatory care: systematic review of diagnostic accuracy studies. Fam Pract 2009;26:10-21.

55. Minnaard MC, de Groot JAH, Hopstaken RM, et al. The added value of $\mathrm{C}$-reactive protein measurement in diagnosing pneumonia in primary care: a meta-analysis of individual patient data. CMAJ 2017;189:E56-E63.

56. Huang Y, Chen R, Wu T, et al. Association between point-of-care CRP testing and antibiotic prescribing in respiratory tract infections: a systematic review and meta-analysis of primary care studies. $\mathrm{Br} \mathrm{J}$ Gen Pract 2013;63:e787-e794.

57. Ting JY, Synnes A, Roberts A, et al. Association Between Antibiotic Use and Neonatal Mortality and Morbidities in Very Low-Birth-Weight Infants Without Culture-Proven Sepsis or Necrotizing Enterocolitis. JAMA Pediatr 2016;170:1181-7.

58. Oliveira CF, Botoni FA, Oliveira CR, et al. Procalcitonin versus C-reactive protein for guiding antibiotic therapy in sepsis: a randomized trial. Crit Care Med 2013;41:2336-43.

59. Barsness KA, Bensard DD, Partrick DA, et al. IL-1beta induces an exaggerated pro- and anti-inflammatory response in peritoneal macrophages of children compared with adults. Pediatr Surg Int 2004;20:238-42.

60. Lawrence RM, Pane CA. Human breast milk: current concepts of immunology and infectious diseases. Curr Probl Pediatr Adolesc Health Care 2007;37:7-36.

61. Sack U, Burkhardt U, Borte M, et al. Age-dependent levels of select immunological mediators in sera of healthy children. Clin Diagn Lab Immunol 1998;5:28-32.

62. Wynn JL, Neu J, Moldawer LL, et al. Potential of immunomodulatory agents for prevention and treatment of neonatal sepsis. J Perinatol 2009;29:79-88 